\title{
Inhibition of the PI3K/Akt signaling pathway reverses sorafenib-derived chemo-resistance in hepatocellular carcinoma
}

\author{
HAO ZHANG ${ }^{*}$, QINGQING WANG ${ }^{*}$, JUN LIU and HAOQIANG CAO \\ Department of General Surgery, The First Hospital of Jiaxing, Jiaxing, Zhejiang 314001, P.R. China
}

Received June 4, 2016; Accepted April 9, 2018

DOI: $10.3892 / \mathrm{ol} .2018 .8536$

\begin{abstract}
Long-term sorafenib treatment triggers resistance to chemotherapy in patients with hepatocellular carcinoma (HCC). In order to investigate the mechanisms of sorafenib resistance in $\mathrm{HCC}$, the aim of the present study was to develop a resistant human liver cell line via long-term exposure to sorafenib. The cytotoxicity cell counting kit- 8 assay was used to evaluate drug sensitivity. Reverse transcription-quantitative polymerase chain reaction and western blotting were used to examine the molecular mechanisms underpinning sorafenib resistance. Migratory and invasive properties in resistant cells were assessed using Transwell assays. The results from the present study revealed that resistant cells became insensitive to sorafenib treatment and exhibited increased migratory and invasive capacities. Activation of the phosphatidylinositol-3-kinase (PI3K)/Akt signaling pathway and epithelial-mesenchymal transition was characteristic of resistant cells. The use of LY294002, a PI3K inhibitor, was able to suppress the activation of Akt and extracellular signal-regulated kinase 1/2, attenuated the migratory and invasive capacities of resistant cells. Data from the present study indicates that inhibition of the PI3K signaling pathway with LY294002 exerts suppressive effects on sorafenib resistance and provides an attractive novel therapeutic regime in patients with advanced HCC.
\end{abstract}

Correspondence to: Dr Hao Zhang, Department of General Surgery, The First Hospital of Jiaxing, 1882 Zhonghuan South Road, Jiaxing, Zhejiang 314001, P.R. China

E-mail: changgung@163.com

*Contributed equally

Abbreviations: HCC, hepatocellular carcinoma; EMT, epithelial-to-mesenchymal cell transition; CCK-8, cell counting kit-8; MMP, matrix metalloproteinases; MAPK, mitogen-activated protein kinase; ERK, extracellular signal-regulated kinase; $\mathrm{IC}_{50}$, half-maximum inhibitory concentration

Key words: hepatocellular carcinoma, sorafenib resistance, epithelial-mesenchymal transition, protein kinase B, ERK

\section{Introduction}

Hepatocellular carcinoma (HCC) is the sixth most common malignancy and the third leading cause of cancer-associated mortality worldwide $(1,2)$. In particular, China has a high prevalence of HCC attributable to significant hepatitis B virus infection rates (3). The majority of patients with HCC present with intrahepatic or distant metastasis at the time of diagnosis, thereby this reduces the possibility of successful surgical resection or transplantation. Therefore, the development of novel therapeutics for the treatment of advanced HCC is crucial.

Sorafenib, the first-line systemic drug approved by the US Food and Drug Administration for advanced hepatocellular carcinoma, is a multi-kinase inhibitor that exerts inhibitory effects on tumor cell proliferation and angiogenesis through the inactivation of serine-threonine kinase Raf-1, which participates in the Ras/Raf/MEK/mitogen-activated protein kinase (MAPK) signaling cascade. Additionally, other receptor tyrosine kinases are also the target of sorafenib including vascular endothelial growth factor receptor, platelet-derived growth factor receptor, and fibroblast grow th factor receptor $(4,5)$. Previously, a series of clinical randomized controlled trials have shown that sorafenib provides a survival benefit compared with placebo therapy $(6,7)$.

However, acquired drug resistance in patients with $\mathrm{HCC}$ is prevalent in patients who are exposed to sorafenib and other chemotherapeutic agents over a long period of time. Thus, further studies examining the molecular mechanisms by which sorafenib resistance occurs in patients with HCC are needed in order to develop novel therapeutic strategies. Previous studies have attempted to address the mechanisms involved in the development of sorafenib resistance. Indeed, Chen et al (8) demonstrated that the activation of phosphoinositide 3-kinase (PI3K)/Akt signaling is associated with acquired resistance to sorafenib in HCC. Furthermore, previous studies have also revealed that epithelial-mesenchymal transition (EMT) is able to render cancer cells not only with drug resistance, but also with invasive and metastatic properties (9). Additionally, it has been suggested that the activation of the PI3K/Akt signaling pathway may promote EMT $(8,10)$. However, whether activation of the PI3K/Akt cascade results in enhanced invasion of sorafenib resistant cells associated with EMT remains unclear.

In the present study, the changes in Huh7 cells with regard to morphology, migration and invasion properties following 
sorafenib treatment were examined. Furthermore, alterations in the PI3K/Akt signaling pathway and activation of EMT following long-term exposure to sorafenib were analyzed. Finally, the effect of a PI3K inhibitor, LY294002, on the viability, migration and invasion of resistant Huh7 cancer cells was investigated.

\section{Materials and methods}

Reagents and antibodies. Sorafenib was purchased from Bayer AG (Leverkusen, Germany). The PI3K inhibitor LY294002 and all antibodies for immunoblotting were obtained from Cell Signaling Technology, Inc. (Danvers, MA, USA), unless specified below.

Cell culture. Huh7, a human HCC cell line, was obtained from the Cell Bank Type Culture Collection of Chinese Academy of Sciences (Shanghai, China). The cells were cultured in Dulbecco's modified Eagle's medium (DMEM; Invitrogen; Thermo Fisher Scientific, Inc., Waltham, MA, USA) containing $10 \%$ fetal bovine serum (FBS; Gibco; Thermo Fisher Scientific, Inc.) in a humidified $5 \% \mathrm{CO}_{2}$ incubator at $37^{\circ} \mathrm{C}$.

Reverse transcription-quantitative polymerase chain reaction $(R T-q P C R)$. Total RNA was extracted using Trizol reagent (Invitrogen; Thermo Fisher Scientific, Inc.), and the concentration of RNA was quantified by SmartSpec Plus spectrophotometer (Bio-rad Laboratories, Inc., Hercules, CA, USA). The first-strand complementary DNA was synthesized using the Prime Script RT reagent kit with gDNA Eraser (Takara Biotechnology Co., Ltd., Dalian, China). qPCR reactions were performed using SYBR Premix Ex Taq (Takara Biotechnology Co., Ltd). Briefly, the amplification protocol was carried out using an initial denaturation at $95^{\circ} \mathrm{C}$ for $30 \mathrm{sec}$, followed by 40 cycles for $5 \mathrm{sec}$ at $95^{\circ} \mathrm{C}, 30 \mathrm{sec}$ at $60^{\circ} \mathrm{C}$. Following the completion of the PCR amplification reaction, the relative expression levels of the gene of interest were analyzed by the $2^{-{ } \Delta \mathrm{Cq}}$ method (11), and the amplification and melting curves were analyzed. All primer sequences (Sangon Biotech, Shanghai, China) used are listed in Table I.

Cell viability assay. The effect of individual treatment agents on cell viability was assessed with the Cell Counting Kit-8 (CCK-8) assay (Dojindo Molecular Technologies, Inc., Kumamoto, Japan) in three replicates. The cells (5,000/well) were seeded in 96-well plates and incubated for $24 \mathrm{~h}$ at $37^{\circ} \mathrm{C}$. The cells were then incubated with $100 \mu \mathrm{l}$ of fresh medium containing indicated concentrations $(0,1,2,4,6,8,10$ and $12 \mu \mathrm{M}$ ) of sorafenib for $48 \mathrm{~h}$ at $37^{\circ} \mathrm{C}$. Finally, $10 \mu \mathrm{l} \mathrm{CCK}-8$ was added to each well for $1 \mathrm{~h}$ at $37^{\circ} \mathrm{C}$, and the optical density was measured at $450 \mathrm{~nm}$ using a microplate reader (Bio-Rad Laboratories, Inc.). The experiments were repeated three times.

Western blotting. Total protein was extracted using ice-cold cell lysis buffer (Beyotime Institute of Biotechnology, Haimen, China) for $30 \mathrm{~min}$. The mixture was centrifuged at $10,000 \mathrm{~g}$ for $15 \mathrm{~min}$ at $4^{\circ} \mathrm{C}$. The upper layer of separation was collected and stored at $-20^{\circ} \mathrm{C}$ until further use. A total of $30 \mu \mathrm{g}$ total protein were separated by $10 \%$ SDS-PAGE, followed by wet transfer
Table I. Primer sequences for reverse transcription-quantitative polymerase chain reaction.

\begin{tabular}{lc}
\hline Gene & Sequence (5'-3') \\
\hline ABCB1 & \\
Forward & \\
Reverse & GTGCTGGTTGCTGCTTACAT \\
ABCC1 & AGCCTATCTCCTGTCGCATT \\
Forward & GCTGTGAAGACCCAGGAGAG \\
Reverse & AAGCACCAGGAAACCACTTG \\
ABCG2 & \\
Forward & ATCCTTCCATCTTGTTCTTGG \\
Reverse & CGTCCCTGCTTAGACATCCT \\
Snail & \\
Forward & \\
Reverse & GTGGTTCTTCTGCGCTACTG \\
Slug & AGGGCTGCTGGAAGGTAAAC \\
Forward & \\
Reverse & GAGCATTTGCAGACAGGTCA \\
\hline
\end{tabular}

ABCB1, ATP binding cassette subfamily B member 1; ABCC1, ATP binding cassette subfamily $\mathrm{C}$ member 1; ABCG2, ATP binding cassette subfamily $\mathrm{G}$ member 2 .

to polyvinylidene difluoride membranes. The membranes were blocked with $5 \%$ skimmed milk powder dissolved in phosphate buffered saline containing $0.1 \%$ Tween-20 (PBS-T) at room temperature for $1 \mathrm{~h}$. The membranes were incubated overnight at $4^{\circ} \mathrm{C}$ with the following primary antibodies: PI3K (cat. no. ab-125633; 1:1,000 dilution; Abcam, Cambridge, UK), phosphorylated (p)-Akt (cat. no. ab-38449; 1:1,000 dilution; Abcam), Akt (cat. no. ab-126580; 1:1,000 dilution; Abcam), p-extracellular signal-regulated kinase (ERK)1/2 (cat. no. 4370; 1:1,000 dilution; Cell Signaling Technology, Inc.), ERK1/2 (cat. no. 4695; 1:2,000 dilution; Cell Signaling Technology, Inc.), phosphatase and tensin homolog, E-cadherin (cat. no. ab-15148; 1:1,000 dilution; Abcam), N-cadherin (cat. no. ab-18203; 1:1,000 dilution; Abcam), Snail (cat. no. 3879; 1:500 dilution; Cell Signaling Technology, Inc.), Slug (cat. no. 9585; 1:500 dilution; Cell Signaling Technology, Inc., Danvers, MA, USA), Vimentin (cat. no. ab-92547; 1:1,000 dilution; Abcam) and GAPDH (cat. no. ab-9485; 1:1,000 dilution; Abcam). After washing three times with PBST, the membranes were incubated with the secondary antibodies (horseradish peroxidase-conjugated anti-rabbit IgG; cat. no. 4414; 1:2,000 dilution; Cell Signaling Technology, Inc.) for $1 \mathrm{~h}$ at room temperature. After repetition of the washing step with PBST, the membranes were visualized using enhanced chemiluminescence western blotting detection reagents (BeyoECL Plus; Beyotime Institute of Biotechnology).

Migration and invasion Transwell assays. Migration and invasion assays were performed using Transwell chambers containing a membrane filter with $8 \mu \mathrm{m}$ pores (Corning Incorporated, Corning, NY, USA) inserted in 24-well plates. The upper chambers were coated with Matrigel (BD Biosciences, San Jose, CA, 
A
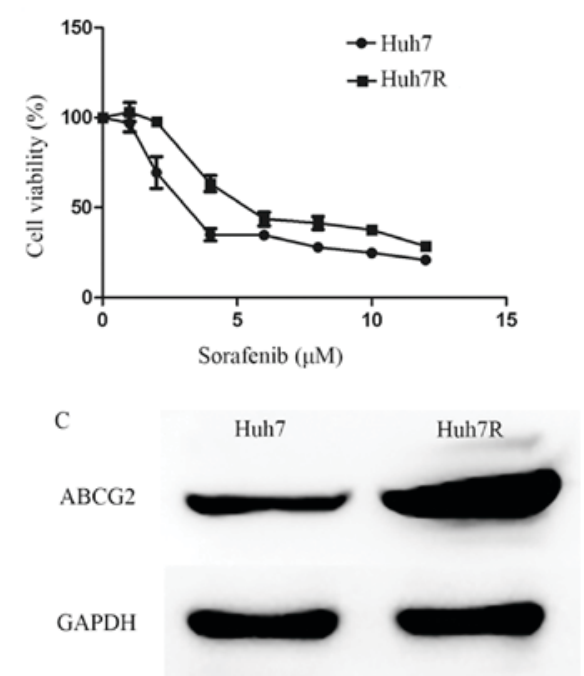

B
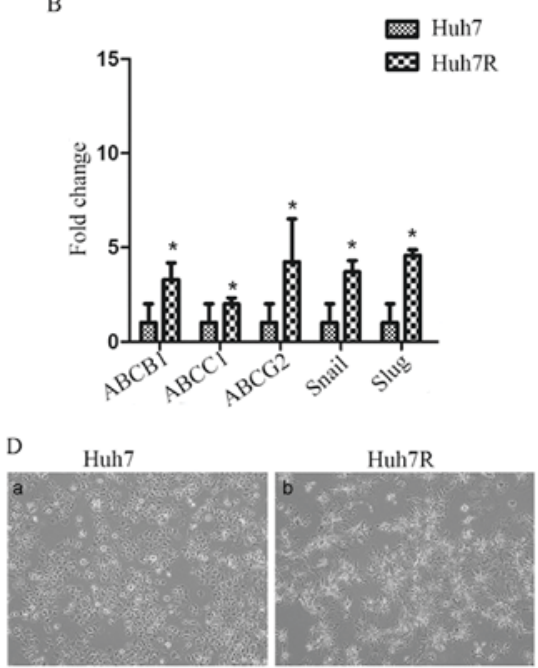

Figure 1. Establishment of a sorafenib-resistant Huh7 cell line. (A) Huh7 and Huh7R cells were cultured with different concentrations of sorafenib (0-12 $\mu$ M), and cell viability was determined using the Cell Counting- 8 assay. (B) Reverse transcription quantitative-polymerase chain reaction analysis of ABCB1, ABCC1, ABCG2, Snail and Slug in Huh7 and Huh7R cells was performed. (C) Western blot analysis of ABCG2 in sorafenib-resistant cells and parental cells. (D) Phase-contrast micrographs of Huh7 and Huh7R (magnification, x100). The relative abundances of target mRNAs were plotted as fold-change compared with the control group. GAPDH served as the loading control. Statistical analysis was performed with Student's t test. * $<<0.05$. ABCB1, ATP binding cassette subfamily B member 1; ABCC1, ATP binding cassette subfamily C member 1; ABCG2, ATP binding cassette subfamily G member 2. All experiments were performed in triplicate. Data are presented as the mean \pm standard deviation.

USA), and the top of each well was seeded with $5 \times 10^{4}$ cells in a total volume of $100 \mu \mathrm{l}$ serum-free DMEM. The lower chamber wells were loaded with $500 \mu \mathrm{l}$ DMEM containing 10\% FBS which served as a chemo-attractant. After $60 \mathrm{~h}$ of incubation, the invaded cells were fixed with $4 \%$ paraformaldehyde for $15 \mathrm{~min}$ at room temperature and then stained with $0.1 \%$ crystal violet for $2 \mathrm{~h}$. The invaded cells were counted under a phase-contrast microscope in five random fields per insert at x100 magnification. A cell migration assay was performed using a similar protocol with the exception that Matrigel was not added to the upper chambers.

Statistical analysis. Data are presented as the mean \pm standard deviation. The one-way analysis of variance or Student's t-test was executed to assess the statistical significance using SPSS version 17.0 software (SPSS, Inc., Chicago, IL, USA) and $\mathrm{P}<0.05$ was considered to indicate a statistically significant difference.

\section{Results}

Characteristics of sorafenib-resistant cells. Through long-term exposure to increasing concentrations of sorafenib, Huh7 cells were successfully transformed a sorafenib-resistant Huh7 cell line (Huh7R) was established. Huh7R cells were less sensitive to sorafenib treatment and their viability was markedly higher compared with that of parental cells when exposed to the same concentration of sorafenib (Fig. 1A). Furthermore, the half-maximum inhibitory concentration $\left(\mathrm{IC}_{50}\right)$ of the resistant cells increased $\sim 1.7$-fold compared with the parental Huh7 cells (Table II). In addition, it was demonstrated that there was an increased level of ABCB1, ABCC1 and ABCG2 mRNA expression (Fig. 1B) and increased levels of $A B C G 2$ protein in the Huh7R cell line (Fig. 1C) compared with Huh7, further
Table II. $\mathrm{IC}_{50}$ of parental and resistant cells following sorafenib treatment.

\begin{tabular}{lc}
\hline Cell line & $\mathrm{IC}_{50}(\mu \mathrm{M})$ \\
\hline Huh7 & 3.7 \\
Huh7-R & 6.4 \\
\hline
\end{tabular}

confirming resistance to sorafenib. Notably, Huh7R cells exhibited microscopically elongated fibroblastic processes and loss of cell-to-cell contacts (Fig. 1D).

Epithelial-mesenchymal transition in sorafenib-resistant cells. Resistant cells underwent morphological changes, which were likely to be associated with EMT (Fig. 1D). To evaluate whether these morphological changes in the resistant cell line Huh7R were associated with EMT, markers characteristic of EMT were tested. Huh7R cells exhibited upregulated mRNA expression of Snail and Slug compared with Huh cells (Fig. 1B). In addition, immunoblotting indicated a marked reduction in the expression of the epithelial marker E-cadherin and a marked upregulation of mesenchymal markers, including Snail, Slug, vimentin and N-cadherin in Huh7R resistant cells compared with control parental cells (Fig. 2). Moreover, it was demonstrated that Huh7R resistant cells expressed expression of matrix metalloproteinase (MMP)-2 and MMP-9 at the protein level (Fig. 2). Together, the present study indicated that EMT was a characteristic of sorafenib-resistant cells.

Enhanced migration and invasion capacities of sorafenib-resistant cells. EMT is an important process, which 


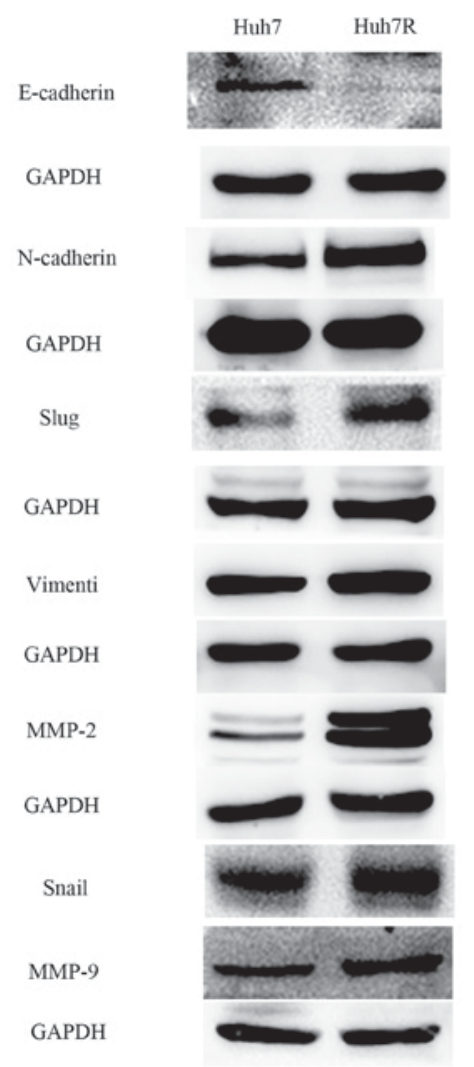

Figure 2. Epithelial-to-mesenchymal transition in sorafenib-resistant cells. Western blot analysis of E-cadherin, N-cadherin, vimentin, Snail, Slug, MMP-2, and MMP-9 to evaluate the levels of protein expression was performed. MMP, matrix metalloproteinase.

results in enhanced invasive and metastatic properties in cancer cells (12). Therefore, Transwell assays were performed in order to evaluate the migration and invasion capacities of Huh7 and Huh7R cells. The data revealed that the migration and invasion of Huh7R cells was significantly increased compared with Huh7 cells (Fig. 3).

Development of sorafenib resistance is associated with activation of the PI3K/Akt signaling pathway. To better understand the molecular mechanisms associated with the development of sorafenib resistance, key molecules within the PI3K/Akt and ERK pathways were investigated. Huh7R resistant cells expressed increased levels of PI3K, p-Akt and p-ERK1/2 compared with their parental Huh7 cells (Fig. 4A). The results from the present study indicate that long-term exposure of $\mathrm{HCC}$ cells to sorafenib may activate the PI3K/Akt and ERK signaling pathways. Based on findings by Chen et al (8), the possibility of inhibiting PI3K with LY294002 in order to reverse sorafenib resistance was investigated. Indeed, it was demonstrated that a combination of different concentrations of sorafenib and a non-toxic dose $(50 \mu \mathrm{M})$ of LY294002 resulted in decreased viability of resistant cells (Fig. 4B) compared with untreated cells. Furthermore, it was observed that the combined treatment caused attenuation of the p-Akt and increased expression of pro-apoptotic proteins, including Bax (Fig. 4C-E) compared with the expression in untreated cells. Furthermore, the activation of Akt and ERK1/2 was suppressed in Huh7R
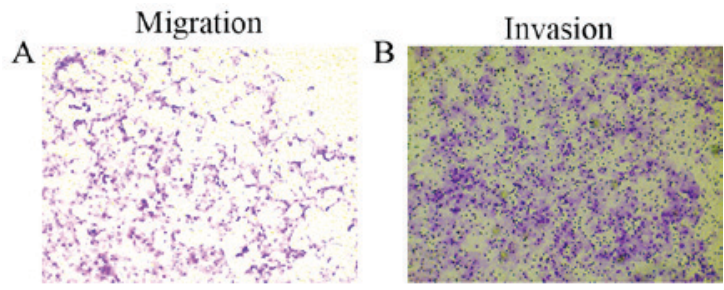

$\mathrm{C}$
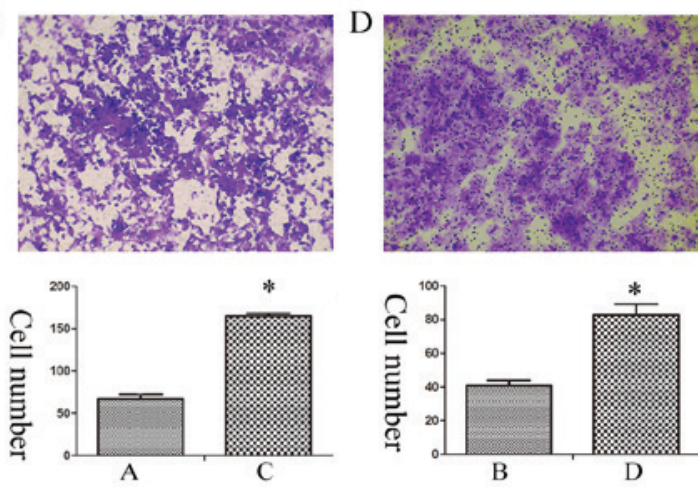

Figure 3. Enhanced migration and invasion of sorafenib-resistant cells Migration and invasion of (A and B) Huh7 and (C and D) Huh7R were examined using Transwell assays, and assessed by counting the number of cells under phase-contrast microscopy (magnification, x100). The bar charts demonstrate the average number of migratory or invasive cells in the repeated experimental groups. Statistical analysis was performed with Student's t-test. ${ }^{*} \mathrm{P}<0.05$.

resistant cells that were treated with LY294002, in a doseand time-dependent manner (Fig. 4C-E).

Enhanced migration and invasion is associated with activation of the PI3K/Akt signaling pathway. To better understand the signaling mechanisms involved in the invasion of Huh7R cells following long-term exposure to sorafenib, the activation of PI3K/Akt and ERK signaling pathways was studied. Treatment of sorafenib resistant cells with the PI3K inhibitor, LY294002, markedly attenuated the activation of Akt (Ser473) and ERK1/2 in a concentration and time-dependent manner. Furthermore, treatment with LY294002 was able to suppress the expression of mesenchymal markers including N-cadherin, vimentin, Snail and Slug, and induce the expression of E-cadherin, which is an epithelial marker (Fig. 5A, B). Furthermore, the treatment of sorafenib-resistant cells suppressed the expression of MMP-2 in a concentration- and time-dependent manner (Fig. 5C, D). In addition, a dose of $50 \mu \mathrm{M}$ LY294002 significantly prevented the increased migratory and invasive abilities observed in sorafenib-resistant Huh7R cells (Fig. 5E).

\section{Discussion}

Sorafenib is the standard first-line systemic therapy with clinically significant survival advantages for patients with advanced HCC. Unfortunately, clinical evidence confirms that the majority of all patients will eventually become resistant to sorafenib treatment and experience disease progression whilst remaining on therapy, consequently limiting the survival benefit of sorafenib treatment. Chow et al (13) demonstrated that long-term exposure to sorafenib increased migratory and invasive abilities of HCC cells in vitro and in vivo, suggesting 
A

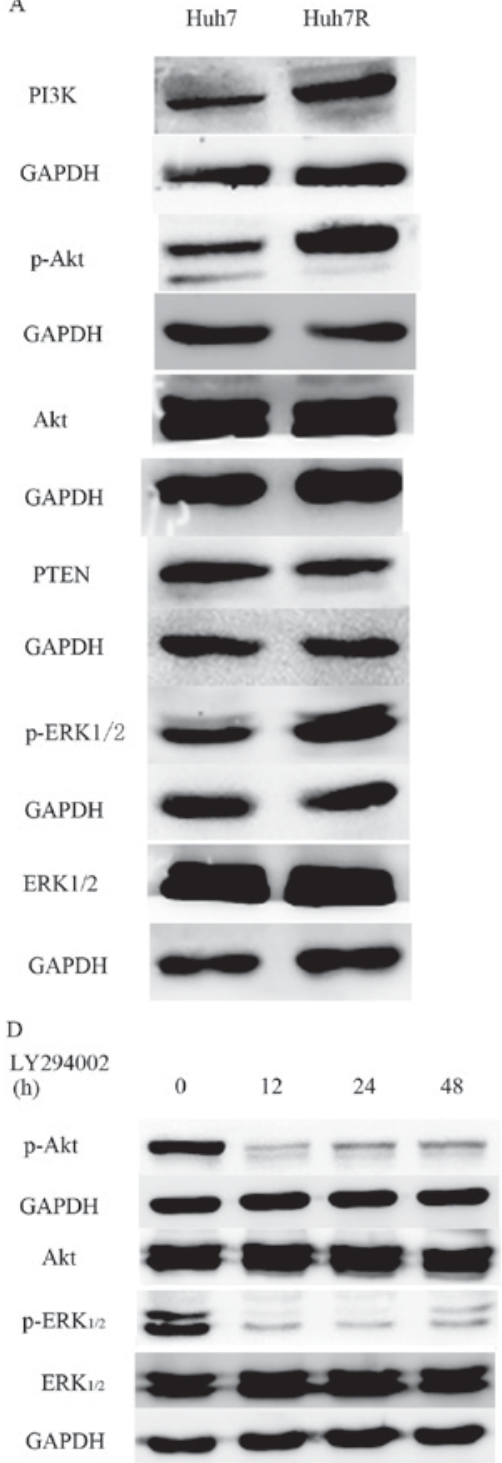

B

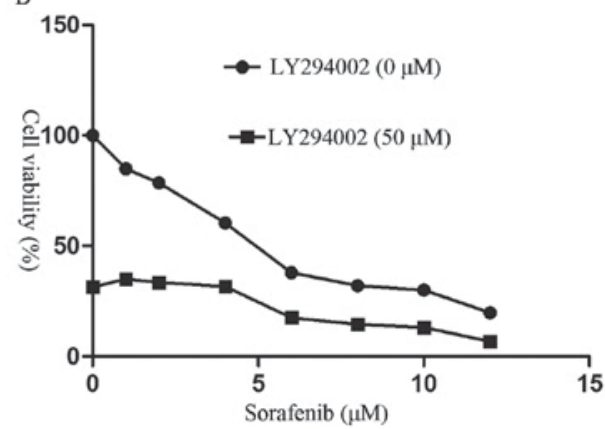

C

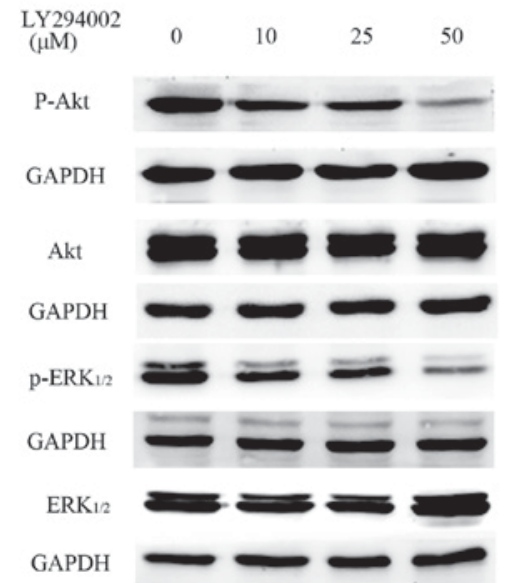

$\mathrm{E}$

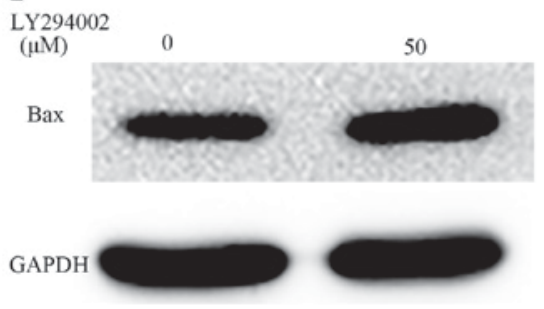

Figure 4. LY294002 reverses sorafenib resistance. (A) Western blot analysis of p-Akt, Akt, p-ERK1/2 and ERK1/2 to detect the protein levels in Huh7 and Huh7R cells. (B) CCK-8 dose-response curves for sorafenib treatment using a concentration of $50 \mu \mathrm{M} \mathrm{LY} 294002$. Western blotting was performed to examine alterations in key signaling pathways in Huh7R cells that were treated with LY294002 at (C) various doses and (D) duration. (E) Western blotting was performed to examine alterations in key apoptotic protein Bax in Huh7R cells that were treated with $50 \mu \mathrm{M}$ LY294002 for $60 \mathrm{~h}$. GAPDH was used as a loading control. ERK, extracellular signal-regulated kinase; CCK-8, Cell Counting Kit-8; p-, phosphorylated.

that an increase in metastatic potential is a characteristic of resistant cells. Thus, it is essential to further elucidate the biological mechanisms involved in the development of chemoresistance and metastasis. In the present study, it was demonstrated that long-term exposure to sorafenib led to drug resistance and was able to increase the migratory and invasive properties of resistant cells in vitro. Importantly, it was also revealed that the activation of the PI3K/Akt signaling pathway was a characteristic of sorafenib-resistant cells.

EMT is a crucial biological process by which malignant tumor cells undergo multiple morphological and biochemical changes including increased cellular motility. This conversion enables tumor cells to acquire drug resistance, and invasive and metastatic properties $(12,14)$, and has indeed attracted considerable attention among researchers. The process of EMT is associated with shorter disease-free survival and poorer prognosis (9). In the present study, Huh7R cells underwent morphological changes associated with EMT, including enhanced migration and invasion. Moreover, downregulation in E-cadherin and upregulation of Snail, Slug, Vimentin and $\mathrm{N}$-cadherin further confirmed that EMT was a feature of Huh7R cells following long-term exposure to sorafenib. Consistent with the data in the present study, Chow et al (13) previously demonstrated that sorafenib-induced EMT promoted migration and invasion in HCC cells. However, the molecular mechanisms involved in the process of EMT have, to date, not been clearly elucidated.

Recently, increasing evidence has indicated that the activation of the PI3K/Akt signaling pathway may play an important role in promoting invasion and metastasis in resistant cells (15). It is, therefore, of significant interest to examine whether sorafenib-induced EMT is attributable to the activation of the PI3K/Akt cascade. It has been demonstrated that the PI3K/Akt and ERK signaling pathways are 

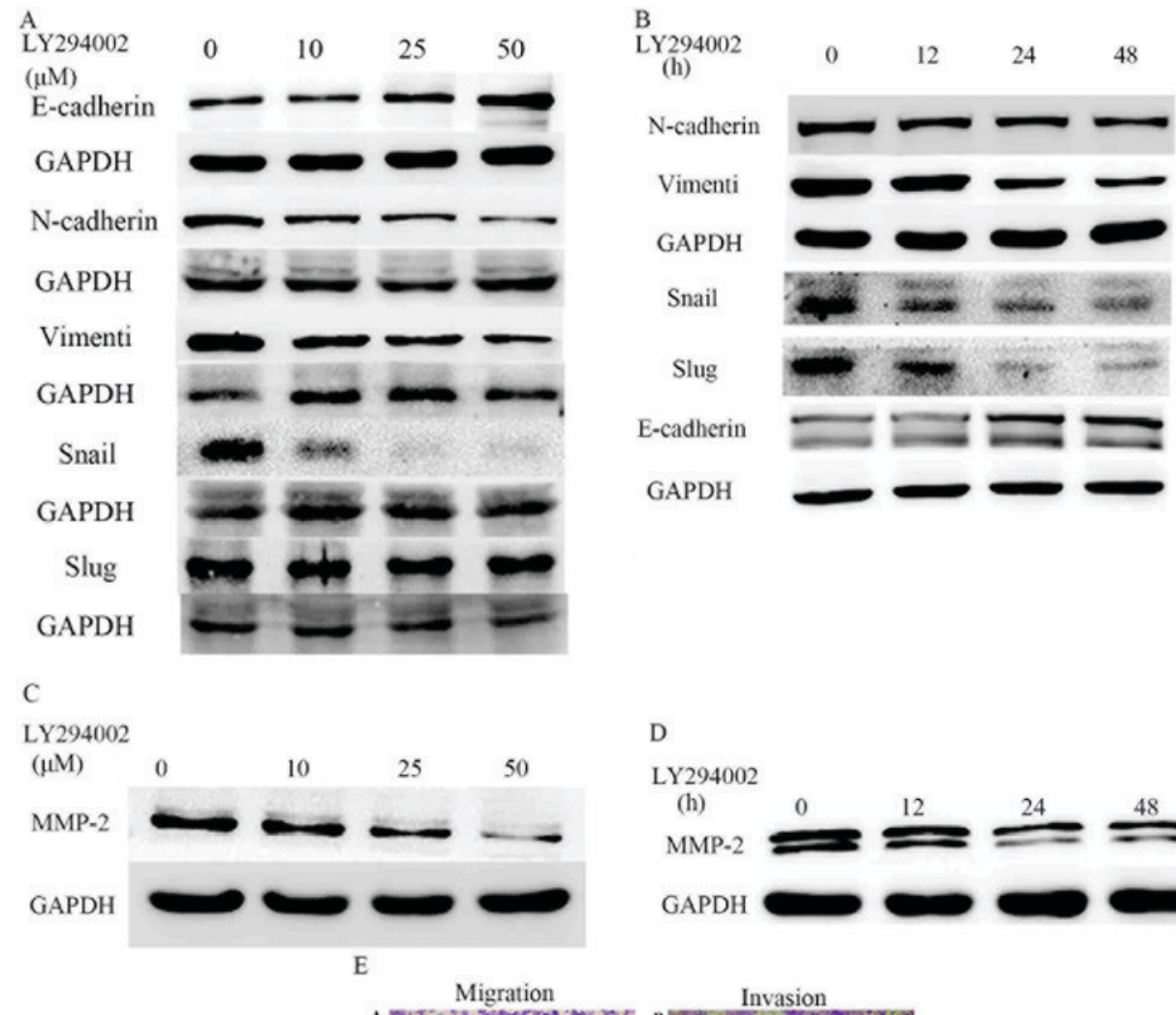

D
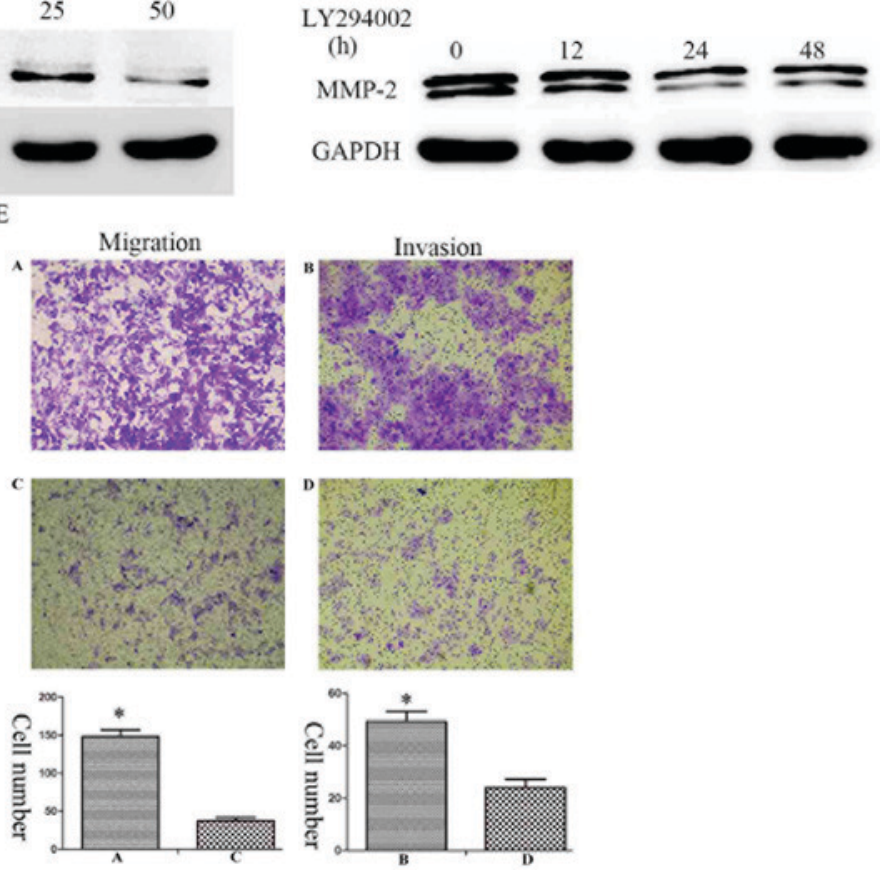

Figure 5. Enhanced migration and invasion is associated with the activation of the PI3K/Akt signaling pathway. Expression of E-cadherin, N-cadherin, Vimentin, Snail and Slug was examined in Huh7-R cells treated with (A) various doses of LY294002 $(0,10,25$ and $50 \mu \mathrm{M})$ and (B) various durations $(0,12$, 24 and $48 \mathrm{~h}$ ) of $50 \mu \mathrm{M} \mathrm{LY294002.} \mathrm{Expression} \mathrm{of} \mathrm{MMP-2} \mathrm{was} \mathrm{examined} \mathrm{in} \mathrm{Huh7-R} \mathrm{cells} \mathrm{treated} \mathrm{with} \mathrm{(C)} \mathrm{various} \mathrm{doses} \mathrm{of} \mathrm{LY294002} \mathrm{(0,} \mathrm{10,} 25$ and 50 $\mu \mathrm{M})$ and (D) various durations $(0,12,24$ and $48 \mathrm{~h}$ ) of $50 \mu \mathrm{M}$ LY294002. (E) Huh7R cells were treated with 0 and $50 \mu \mathrm{M}$ LY294002. The cells in panels (A) and (B) were treated with $0 \mu \mathrm{M} \mathrm{LY} 294002$, and the cells in panels (C) and (D) were treated with $50 \mu \mathrm{M} \mathrm{LY} 294002$. Migration and invasion were examined using Transwell assays. The bar charts demonstrate the average number of migratory or invasive cells in the experimental groups. Statistical analysis was performed with Student's t-test. ${ }^{*} \mathrm{P}<0.05$.

involved in the process of EMT (16). Thus, analysis of the status of the PI3K/Akt and ERK1/2 pathways was examined in the present study. Notably, it was demonstrated that inhibition of the PI3K/Akt signaling pathway with LY294002, a PI3K inhibitor, had an effect on ERK and thus suppressed the initiation of EMT. Together, these data suggest not only a crosstalk between the PI3K/Akt and ERK1/2 signaling pathways, but also that EMT may directly or indirectly regulate transcription factors associated with these pathways, including Snail and Slug.

The MMP-2 and MMP-9 have been associated with migration and invasion of resistant cells. This phenomenon may be regulated by the activation of the PI3K/Akt and MAPK signaling pathways (17). The data of the present study indicated that the PI3K/Akt cascade may directly or indirectly regulate the properties of migration and invasion in sorafenib-resistant cells via MMP-2 and MMP-9. Taken together, the results suggest that the PI3K/Akt signaling pathway contributes a pivotal role in directly or indirectly regulating the migratory and invasive capacities of sorafenib-resistant cells via EMT and MMPs.

The PI3K/Akt signaling cascade is known to be an important signal transduction pathway involved in hepatocarcinogenesis. Multiple studies have reported that the activation 
of the PI3K/Akt signaling pathway induces chemoresistance in HCC cells (18-20). Meanwhile, the MAPK signaling pathway is a major target of sorafenib therapy. However, several studies have also clearly indicated that activation of the MAPK cascade is associated with drug resistance (21-23). Concomitant with the present study, overexpression of $\mathrm{p}-\mathrm{Akt}$ and p-ERK1/2 results in sorafenib resistance (24). The PI3K inhibitor LY294002 is able to inhibit the activation of ERK, which indicates that ERK activation may be induced by the PI3K/Akt signaling pathway. In the present study, it was also demonstrated that attenuation of the PI3K/Akt signaling pathway using LY294002 was able to induce the expression of apoptotic proteins, including Bax, and cell viability was also decreased. These data suggested that the PI3K/Akt signaling pathway may have an important role in sorafenib resistance.

In conclusion, the results from the present study highlight the activation of the PI3K/Akt signaling pathway as a key event in the acquisition of resistance and the enhancement of invasive properties in sorafenib-resistant cells. Furthermore, the present study indicates that combined treatment with sorafenib and LY294002 may reduce resistance to sorafenib and prevent metastasis in patients with HCC, providing an attractive novel therapeutic regime in patients with advanced HCC.

\section{Acknowledgements}

Not applicable.

\section{Funding}

This study was supported by grants from Jiaxing Science and Technology Projects (grant no. 2013AY21042-5), Jiaxing Science and Technology innovation team projects (grant no. 2013-03), and major projects of Zhejiang Province on the transformation of the appropriate technical achievements of primary health care (grant no. 2013T301-12 and 2013T301-15).

\section{Availability of data and materials}

The datasets used and/or analyzed during the current study are available from the corresponding author on reasonable request.

\section{Author contributions}

HZ performed the preliminary studies on which this work is based, conceived the study, collected and analysed data and drafted the manuscript. QW performed the RT-PCR and western-blot analysis and was also involved in the study design and editing of the manuscript. JL and HC were involved in the experiment planning, the drafting of the manuscript and for revising it critically for content and general supervision of the project. All the authors have read and approved the final manuscript.

\section{Ethics approval and consent to participate}

Animal experiments were performed in accordance with the animal guidelines of the First Hospital of Jiaxing Ethics Review Committee for Animal Experimentation.

\section{Consent for publication}

Not applicable.

\section{Competing interests}

The authors declare that they have no competing interests.

\section{References}

1. Forner A, Llovet JM and Bruix J: Hepatocellular carcinoma. Lancet 379: 1245-1255, 2012.

2. Torre LA, Bray F, Siegel RL, Ferlay J, Lortet-Tieulent J and Jemal A: Global cancer statistics, 2012. CA Cancer J Clin 65: 87-108, 2015.

3. Chen W, Zheng R, Baade PD, Zhang S, Zeng H, Bray F, Jemal A, Yu XQ and He J: Cancer statistics in China, 2015. CA Cancer J Clin 66: 115-132, 2016.

4. Liu L, Cao Y, Chen C, Zhang X, McNabola A, Wilkie D, Wilhelm S, Lynch $M$ and Carter C: Sorafenib blocks the $\mathrm{RAF/MEK/ERK} \mathrm{pathway,} \mathrm{inhibits} \mathrm{tumor} \mathrm{angiogenesis,} \mathrm{and}$ induces tumor cell apoptosis in hepatocellular carcinoma model PLC/PRF/5. Cancer Res 66: 11851-11858, 2006.

5. Chen S, Wang Y, Ruan W, Wang X and Pan C: Reversing multidrug resistance in hepatocellular carcinoma cells by inhibiting extracellular signal-regulated kinase/mitogen-activated protein kinase signaling pathway activity. Oncol Lett 8: 2333-2339, 2014.

6. Cheng AL, Kang YK, Chen Z, Tsao CJ, Qin S, Kim JS, Luo R, Feng J, Ye S, Yang TS, et al: Efficacy and safety of sorafenib in patients in the Asia-Pacific region with advanced hepatocellular carcinoma: A phase III randomised, double-blind, placebo-controlled trial. Lancet Oncol 10: 25-34, 2009.

7. Rimassa L and Santoro A: Sorafenib therapy in advanced hepatocellular carcinoma: The SHARP trial. Expert Rev Anticancer Ther 9: 739-745, 2009.

8. Chen KF, Chen HL, Tai WT, Feng WC, Hsu CH, Chen PJ and Cheng AL: Activation of phosphatidylinositol 3-kinase/Akt signaling pathway mediates acquired resistance to sorafenib in hepatocellular carcinoma cells. J Pharmacol Exp Ther 337: 155-161, 2011.

9. Ombrato L and Malanchi I: The EMT universe: Space between cancer cell dissemination and metastasis initiation. Crit Rev Oncog 19: 349-361, 2014.

10. Wang H, Xu L, Zhu X, Wang P, Chi H and Meng Z: Activation of phosphatidylinositol 3-kinase/Akt signaling mediates sorafenib-induced invasion and metastasis in hepatocellular carcinoma. Oncol Rep 32: 1465-1472, 2014.

11. Livak KJ and Schmittgen TD: Analysis of relative gene expression data using real-time quantitative PCR and the 2(-Delta Delta C(T)) method. Methods 25: 402-408, 2001.

12. van Malenstein H, Dekervel J, Verslype C, Van Cutsem E, Windmolders P, Nevens F and van Pelt J: Long-term exposure to sorafenib of liver cancer cells induces resistance with epithelial-tomesenchymal transition, increased invasion and risk of rebound growth. Cancer Lett 329: 74-83, 2013.

13. Chow AK, Ng L, Lam CS, Wong SK, Wan TM, Cheng NS, Yau TC, Poon RT and Pang RW: The Enhanced metastatic potential of hepatocellular carcinoma (HCC) cells with sorafenib resistance. PLoS One 8: e78675, 2013.

14. Mir N, Jayachandran A, Dhungel B, Shrestha R and Steel JC: Epithelial-to-mesenchymal transition: A mediator of sorafenib resistance in advanced hepatocellular carcinoma. Curr Cancer Drug Targets 17: 698-706, 2017.

15. Xu W, Yang $\mathrm{Z}$ and Lu N: A new role for the PI3K/Akt signaling pathway in the epithelial-mesenchymal transition. Cell Adh Migr 9: 317-324, 2015.

16. Wen W, Ding J, Sun W, Fu J, Chen Y, Wu K, Ning B, Han T, Huang L, Chen C, et al: Cyclin G1-mediated epithelialmesenchymal transition via phosphoinositide 3-kinase/Akt signaling facilitates liver cancer progression. Hepatology 55: 1787-1798, 2012.

17. Wu YJ, Neoh CA, Tsao CY, Su JH and Li HH: Sinulariolide suppresses human hepatocellular carcinoma cell migration and invasion by inhibiting matrix metalloproteinase-2/-9 through MAPKs and PI3K/Akt signaling pathways. Int J Mol Sci 16: 16469-16482, 2015. 
18. Kunter I, Erdal E, Nart D, Yilmaz F, Karademir S, Sagol O and Atabey N: Active form of AKT controls cell proliferation and response to apoptosis in hepatocellular carcinoma. Oncol Rep 31: 573-580, 2014.

19. Dong J, Zhai B, Sun W, Hu F, Cheng $\mathrm{H}$ and $\mathrm{Xu}$ J: Activation of phosphatidylinositol 3-kinase/AKT/snail signaling pathway contributes to epithelial-mesenchymal transition-induced multi-drug resistance to sorafenib in hepatocellular carcinoma cells. PLoS One 12: e0185088, 2017.

20. Zhang PF, Li KS, Shen YH, Gao PT, Dong ZR, Cai JB, Zhang C, Huang XY, Tian MX, Hu ZQ, et al: Galectin-1 induces hepatocellular carcinoma EMT and sorafenib resistance by activating FAK/PI3K/AKT signaling. Cell Death Dis 7: e2201, 2016.

21. Xu Y, Huang J, Ma L, Shan J, Shen J, Yang Z, Liu L, Luo Y, Yao C and Qian C: MicroRNA-122 confers sorafenib resistance to hepatocellular carcinoma cells by targeting IGF-1R to regulate RAS/RAF/ERK signaling pathways. Cancer Lett 371: 171-181,2016.
22. Chen W, Wu J, Shi H, Wang Z, Zhang G, Gao Y, Jiang C and Ding Y: Hepatic stellate cell coculture enables sorafenib resistance in Huh7 cells through HGF/c-Met/Akt and Jak2/Stat3 pathways. Biomed Res Int 2014: 764981, 2014.

23. Li QL, Gu FM, Wang Z, Jiang JH, Yao LQ, Tan CJ, Huang XY, Ke AW, Dai Z, Fan J and Zhou J: Activation of PI3K/AKT and MAPK pathway through a PDGFR $\beta$-dependent feedback loop is involved in rapamycin resistance in hepatocellular carcinoma. PLoS One 7: e33379, 2012.

24. Xu J, Zheng L, Chen J, Sun Y, Lin H, Jin RA, Tang M, Liang X and Cai X: Increasing AR by HIF-2 $\alpha$ inhibitor (PT-2385) overcomes the side-effects of sorafenib by suppressing hepatocellular carcinoma invasion via alteration of pSTAT3, pAKT and pERK signals. Cell Death Dis 8: e3095, 2017. 